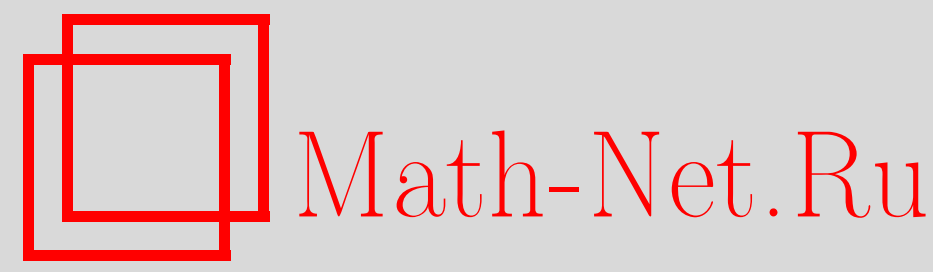

Н. И. Дубровин, Формальные суммы и степенные ряды над группой, Матем. сб., 2000, том 191, номер 7, 13-30

DOI: https://doi.org/10.4213/sm490

Использование Общероссийского математического портала Math-Net.Ru подразумевает, что вы прочитали и согласны с пользовательским соглашением

http: //www.mathnet.ru/rus/agreement

Параметры загрузки:

IP : 3.85 .5 .30

26 апреля 2023 г., $14: 28: 28$ 


\title{
Формальные суммы и степенные ряды над группой
}

\begin{abstract}
Изучаются формальные суммы над группой как алгебраическая система с покомпонентной операцией сложения и частичной операцией свертки “*”. В случае, когда группа правоупорядочена, определяется и изучается модуль формальных степенных рядов - формалњные суммы с вполне упорядоченным носителем. Особое внимание уделяется системам формальных степенных рядов (носитель вполне упорядочен по возрастанию), которые образуют $L$-базис, т.е. для которых любой другой формальньй степенной ряд однозначно разложим по этой системе. $L$-базисы связаны с автоморфизмами модуля формальных рядов, обладающими естественньми свойствами монотонности и $\sigma$-линейности. Исследуются также соотношения $\gamma * \beta=0$ и $\gamma * \beta=1$. Заметим, что в случае линейно упорядоченной групшы система формальных степенных рядов образует тело с нормированием (Мальцев-Нейман, 1948-1949 гг.).

Библиографол: 6 названий.
\end{abstract}

\section{Введение}

Пусть $F$ - тело, $G$ - группа, $U$ - подгруппа группы $G$, а $K$ - кольцо, содержащее групповое кольцо $F U$ в качестве подкольца. Обозначим через $G \searrow U$ множество представителей левых смежных классов $g U(g \in G)$. Считаем в дальнейшем, что $1_{G} \in G \searrow U$. Для любого элемента $g \in G$ через $\bar{g}$ будем обозначать представитель смежного класса $g U$, принадлежащий $G \searrow U$.

Рассмотрим множество $\mathscr{F}(G \searrow U, K)$ всех формальных сумм:

$$
\gamma=\sum_{h \in G \searrow U}^{\prime} h k_{h} \quad\left(k_{h} \in K\right) .
$$

Штрих около знака суммирования указывает, что (1) - именно формальная сумма, т.е. иной способ записи отображения $G \searrow U \rightarrow K$. Без штриха знак суммирования будет употребляться в обычном смысле. Если $U$ - единичная группа, то $G \searrow U=G, K=F$ и $\mathscr{F}(G \searrow U, K)$ обозначаем как $\mathscr{F}(G, F)$. Ясно, что множество формальных сумм $\mathscr{F}(G \searrow U, K)$ несет на себе структуру $(F-K)$-бимодуля относительно покомпонентных операций сложения и умножения. Далее, множество формальных сумм $\mathscr{F}(G \searrow U, K)$ несложно превратить в левый $G$-модуль и тем самым в $(F G-K)$-бимодуль. Более того, можно определить частичную операцию свертки

$$
\mathscr{F}(G, F) * \mathscr{F}(G \searrow U, K) \rightarrow \mathscr{F}(G \searrow U, K)
$$

Работа вьполнена при поддержке совместного фонда Р $\Phi$ И-Германия (грант № 98-01-04110).

(C) н.и. Дувровин 2000 
Таким образом, мы получаем частичную бинарную операцию на $\mathscr{F}(G, F)$ и очень интересную алгебраическую систему $(\mathscr{F}(G, F),+, *, 0,1)$. В случае, когда $G$ - линейно упорядоченная группа, эта система содержит тело формальных рядов Мальцева-Неймана (см. [1]) как подсистему.

Работа преследует две цели. Первая - изучение систем формальных сумм в $\mathscr{F}(G \searrow U, K)$, которые являются базисами в том же смысле, в каком и система элементов $G \searrow U$ (см. разложение (1)). Вторая цель - изучение соотношений $\gamma * \beta=0$ и $\gamma * \beta=1$ в $\mathscr{F}(G, F)$ при некоторых ограничениях на формальные суммы $\gamma$ и $\beta$. Эти ограничения будут связаны с наличием в групше $G$ конуса $P$, т.е. такого подмоноида, что $P \cup P^{-1}=G$. Если же, кроме того, $P \cap P^{-1}=\{1\}$, то $P$ назовем чистым конусом (подробнее о конусах см. [2]).

Результаты работы изложены в шести теоремах и их следствиях; основным результатом является теорема 2.

Смысл обозначений $F, G, U, P, K$ сохраняется на протяжении всей работы. Через 1 обозначаем нейтральный элемент относительно умножения в любой алгебраической системе. Если задан конус $P$, то $U$ всегда обозначает группу его обратимых элементов, а

$$
g_{1} \geqslant g_{2} \Longleftrightarrow g_{1}^{-1} g_{2} \in P, \quad g_{1} \geqslant_{r} g_{2} \Longleftrightarrow g_{2} g_{1}^{-1} \in P
$$

- левъй и правый линейные предпорядки, ассоциированные с конусом Р. Это значит, что условие $g \leqslant h$ влечет неравенство $z g \leqslant z h$, а из $g \leqslant r h$ следует $g z \leqslant r h z$ для любого $z \in G$. Если $P$ - чистый конус, то $U=\{1\}$ и отношение

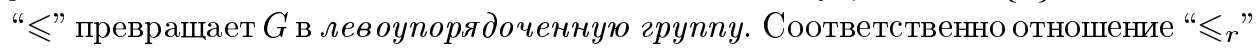
превращает $G$ в правоупорядоченную групnу (см. [3; гл. $5, \S 4]$ ). Как правило, мы будем иметь дело с левым предпорядком, и если нет специальной оговорки, то именно этот предпорядок и будет подразумеваться.

\section{§1. Основные определения и свойства}

1.1. Суммируемые системы. Прежде всего, договоримся через $(\gamma)_{h}$ обозначать коэффициент $k_{h}$ для формальной суммы (1). Множество $\left\{h \in G \searrow U: k_{h} \neq 0\right\}$ назовем носителем суммы $\gamma$ и обозначим его через supp $\gamma$.

ОПРЕДЕЛЕНИЕ 1. Пусть $\left\{\gamma_{i}: i \in I\right\}$ - семейство формальных сумм. Назовем это семейство $\mathscr{F}$-суммируемым, элемент $\gamma \in \mathscr{F}(G \backslash U, K)$ - $\mathscr{F}$-суммой этого семейства и будем писать $\gamma=\sum_{i \in I}^{\mathscr{F}} \gamma_{i}$, если имеет место равенство $(\gamma)_{g}=$ $\sum_{i \in I}\left(\gamma_{i}\right)_{g}$ в кольце $K$ для любого $g \in G \searrow U$ (в частности, это означает, что последняя сумма содержит лишь конечное число ненулевых слагаемых).

Достаточно очевидно

ПРЕДЛОЖЕНИЕ 1. Система формальных сумм $\left\{\gamma_{i}: i \in I\right\} \mathscr{F}$-суммируема тогда и только тогда, когда для любой $\mathbb{N}$-последовательности $i_{1}, i_{2}, i_{3}, \ldots$ попарно различных индексов $и$ любого набора әлементов $g_{j} \in \operatorname{supp} \gamma_{i_{j}}$ $(j=1,2,3, \ldots)$ найдется пара различных натуральных чисел $j, k$ такая, что $g_{j} \neq g_{k}$.

Отметим свойство линейности $\mathscr{F}$-суммы. 
ПРЕДЛОЖЕНИЕ 2. Пусть $\left\{\gamma_{i}: i \in I\right\} u\left\{\delta_{i}: i \in I\right\}-\mathscr{F}$-суммируемье семейства, $a \in F, k \in K-$ произвольные әлементы. Тогда семейство $\left\{a \gamma_{i} k+a \delta_{i} k: i \in I\right\}$ также $\mathscr{F}$-суммируемо $и$

$$
\sum_{i \in I}^{\mathscr{F}}\left(a \gamma_{i} k+a \delta_{i} k\right)=a\left(\sum_{i \in I}^{\mathscr{F}} \gamma_{i}\right) k+a\left(\sum_{i \in I}^{\mathscr{F}} \delta_{i}\right) k
$$

ДокАЗАТЕЛЬСтво. Пусть элемент $h \in G \searrow U$ произволен. Имеем:

$$
\sum_{i \in I}\left(a \gamma_{i} k+a \delta_{i} k\right)_{h}=a\left(\sum_{i \in I}\left(\gamma_{i}\right)_{h}\right) k+a\left(\sum_{i \in I}\left(\delta_{i}\right)_{h}\right) k
$$

В частности, первая сумма содержит лиш конечное число ненулевых слагаемых. Тогда семейство $\left\{a \gamma_{i} k+a \delta_{i} k\right\}$ будет $\mathscr{F}$-суммируемым и

$$
\begin{aligned}
\left(\sum_{i \in I}^{\mathscr{F}}\left(a \gamma_{i} k+a \delta_{i} k\right)\right)_{h} & =\sum_{i \in I}\left(a \gamma_{i} k+a \delta_{i} k\right)_{h}=a\left(\sum_{i \in I}\left(\gamma_{i}\right)_{h}\right) k+a\left(\sum_{i \in I}\left(\delta_{i}\right)_{h}\right) k \\
& =a\left(\sum_{i \in I}^{\mathscr{F}} \gamma_{i}\right)_{h} k+a\left(\sum_{i \in I}^{\mathscr{F}} \delta_{i}\right)_{h} k \\
& =a\left(\sum_{i \in I}^{\mathscr{F}} \gamma_{i}+\sum_{i \in I}^{\mathscr{F}} \delta_{i}\right)_{h} k .
\end{aligned}
$$

Отсюда следует равенство $\mathscr{F}$-сумм.

Элемент $g$ группы $G$ можно записать в виде $g=\bar{g}\left(\bar{g}^{-1} g\right)$. В связи с этим формальную сумму, у которой коэффициент $k_{\bar{g}}$ равен $\bar{g}^{-1} g \in U \subseteq K$, а остальнье коэффициенты - нули, будем обозначать также символом $g$. Из определения 1 следует, что система формальных сумм $\gamma_{h}=h(h \in G \searrow U) \mathscr{F}$-суммируема. Более того, для любого $g \in G$ и для любого набора коэффициентов $k_{h} \in K$ система $\left\{(g h) k_{h}: h \in G \searrow U\right\}$ также будет $\mathscr{F}$-суммируемой. Действительно, если $h_{1}, h_{2} \in G \searrow U-$ разные элементы, то $h_{1} U \neq h_{2} U$, откуда $g h_{1} U \neq g h_{2} U$, т.е. $\overline{g h}_{1} \neq \overline{g h}_{2}$, и остается применить предложение 1 . Это позволяет ввести на множестве формальных сумм структуру левого $G$-модуля:

$$
g\left(\sum_{h \in G \searrow U}^{\prime} h k_{h}\right)=\sum_{h \in G \searrow U}^{\mathscr{F}}(g h) k_{h} \quad\left(k_{h} \in K\right) .
$$

Легко проверяются аксиомы $G$-модуля:

$$
g\left(\gamma_{1}+\gamma_{2}\right)=g \gamma_{1}+g \gamma_{2}, \quad\left(g_{1} g_{2}\right) \gamma=g_{1}\left(g_{2} \gamma\right)
$$

для любых $g, g_{1}, g_{2} \in G, \gamma, \gamma_{1} \gamma_{2} \in \mathscr{F}(G \searrow U, K)$. Кроме того, $g(\gamma k)=(g \gamma) k$ для любого $k \in K$. Следовательно, $\mathscr{F}(G \searrow U, K)-(G-K)$-модуль. По линейности распространим операцию (3) на элементы из группового кольца $F G$. Для этого ненулевой элемент $r \in F G$ запишем в виде

$$
r=a_{1} g_{1}+a_{2} g_{2}+\cdots+a_{n} g_{n} \in F G
$$


где все $a_{i} \in F$ ненулевые, $g_{i} \in G$ и $g_{i} \neq g_{j}$ при $i \neq j(1 \leqslant i, j \leqslant n)$. Тогда по определению

$$
r \gamma=\sum_{i=1}^{n} a_{i} g_{i} \gamma
$$

Тем самьм, $\mathscr{F}(G \searrow U, K)$ превращается в $(F G-K)$-бимодуль. Усилим свойство линейности.

ПрЕДЛОЖЕНИЕ 3. Если $\left\{\gamma_{i}: i \in I\right\}-\mathscr{F}$-суммируемое семейство, а $r \in F G$, то семейство $\left\{r \gamma_{i}\right\}$ такэе $\mathscr{F}$-суммируемо и

$$
r \sum_{i \in I}^{\mathscr{F}} \gamma_{i}=\sum_{i \in I}^{\mathscr{F}} r \gamma_{i}
$$

ДокАЗАТЕльство. Сначала рассмотрим случай $r=g \in G$. Для любого элемента $h \in G \searrow U$ имеет место равенство $\left(g \gamma_{i}\right)_{h}=\left(\gamma_{i}\right) \frac{}{g^{-1} h}$. Следовательно, сумма $\sum_{i \in I}\left(g \gamma_{i}\right)_{h}$ определена, и поэтому система $\left\{g \gamma_{i}\right\}$ суммируема. Равенство (5) проверяется непосредственно.

Пусть теперь элемент $r$ задан как в (4). Тогда, применяя предложение 2 , получаем:

$$
\sum_{i \in I}^{\mathscr{F}} r \gamma_{i}=\sum_{i \in I}^{\mathscr{F}} \sum_{k=1}^{n} a_{k} g_{k} \gamma_{i}=\sum_{k=1}^{n} a_{k} g_{k} \sum_{i \in I}^{\mathscr{F}} \gamma_{i}=r \sum_{i \in I}^{\mathscr{F}} \gamma_{i}
$$

Обозначим через $1 \mathscr{F}$ формальную сумму из $\mathscr{F}(G \searrow U, K)$, у которой коэффициент при $1 \in G \searrow U$ равен единице, а остальные коэффициенты - нули.

ПРЕДЛОЖЕНИЕ 4. Отображение $r \rightarrow r \cdot 1_{\mathscr{F}}(r \in F G)$ задает вложение

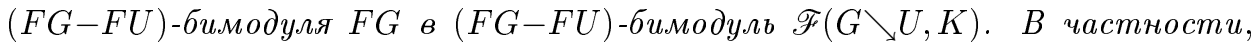
$\mathscr{F}(G \searrow U, K)-$ точный левый $F G$-модуль.

ДокАЗАтЕЛьство. Пусть $r \in F G$ имеет вид (4). Тогда

$$
r \cdot 1_{\mathscr{F}}=\sum_{h \in G \searrow U}^{\prime} h\left(\sum_{1 \leqslant i \leqslant n ; \bar{g}_{i}=h} a_{i} h^{-1} g_{i}\right)
$$

Сумма в круглых скобках не равна нулю, например, для $h=\bar{g}_{1}$. Отсюда $r \cdot 1_{\mathscr{F}} \neq 0$ и инъективность доказана.

Теперь предположим, что в группе $G$ имеется конус $P$ и $U-$ группа его обратимых элементов. Легко проверяется

Лемма 1. Пусть $g_{1}, g_{2} \in G$. Тогда

а) отношение " $\leqslant$ " - линейный порядок на множестве $G \searrow U$;

б) если $g_{1} \leqslant g_{2}$, mo $\bar{g}_{1} \leqslant \bar{g}_{2}$;

в) если $g_{1} \leqslant g_{2}$, то $g g_{1} \leqslant g g_{2}$ для любого $g \in G$, то жсе верно для строгих неравенств;

г) $\bar{g}_{1} \bar{g}_{2}=\bar{g}_{1}{ }_{2}$. 
Пусть, как и ранее, $\gamma=\sum_{h \in G \searrow U}^{\prime} h k_{h}$. Может так случиться, что носитель этой суммы имеет наименьший элемент $h_{0}$. Тогда $h_{0}$ назовем нормой суммы $\gamma$ и обозначим ее через $v(\gamma)$. Однородное слагаемое $h_{0} k_{h_{0}}$ суммы $\gamma$ обозначаем в этом случае через $\partial \gamma$. Ясно, что это однородное слагаемое характеризуется свойством $\operatorname{supp}(\gamma-\partial \gamma)>v(\gamma)$. Неравенство $S_{1}>S_{2}$ для подмножеств $S_{1}, S_{2}$ какого-либо линейно упорядоченного множества здесь и далее будем использовать в том смысле, что любой элемент из левой части строго больше любого элемента из правой части. $\mathrm{K}$ линейно упорядоченному множеству $G \searrow U$ присоединим символ $\infty$, считая, что $h<\infty$ для любого $h \in G \searrow U$ и $v(0)=\infty$.

ОПРЕДЕЛЕНИЕ 2. Формальную сумму $\gamma$ назовем формальным степенным рядом, если множество supp $\gamma$ вполне упорядочено по возрастанию, т.е. любое подмножество этого множества имеет наименьший элемент. Множество всех формальных степенных рядов обозначим через $L$.

ПРЕДЛОЖЕНИЕ 5. Множество $L$ является $(F G-K)$-подбимодулем бимодуля $\mathscr{F}(G \searrow U, K)$.

ДокАЗАтЕльство. Утверждение следует из соотношений, справедливых для любых формальных сумм $\gamma_{1}, \gamma_{2}, \gamma$ и для любых элементов $h \in G, k \in K$ :

$$
\begin{gathered}
\operatorname{supp}\left(\gamma_{1}+\gamma_{2}\right) \subseteq \operatorname{supp} \gamma_{1} \cup \operatorname{supp} \gamma_{2} \\
\operatorname{supp}(g \gamma)=\{\overline{g h}: h \in \operatorname{supp} \gamma\}, \quad \operatorname{supp}(\gamma k) \subseteq \operatorname{supp} \gamma
\end{gathered}
$$

Предположим, что мы имеем систему формальных степенных рядов $\left\{\gamma_{i} \in L\right.$ : $i \in I\}$. На первый взгляд кажется, что суммируемость этой системы в $L$ следует определить как $\mathscr{F}$-суммируемость плюс принадлежность $\mathscr{F}$-суммы подбимодулю $L$. Это определение страдало бы по крайней мере одним сушественньм недостатком: для набора элементов $k_{i} \in K$ система $\left\{\gamma_{i} k_{i}\right\}$ могла быть уже не суммируемой в $L$. Действительно, если $h^{2}<h$ для некоторого $h \in G$, то система $\left\{h^{n}-h^{n+1}: n \in \mathbb{N}\right\} \mathscr{F}$-суммируема и $\mathscr{F}$-сумма, равная $h$, принадлежит $L$. Однако система $\left\{\left(h^{n}-h^{n+1}\right)(-1)^{n}: n \in \mathbb{N}\right\}$ имеет сумму $-h+2 h^{2}-2 h^{3}-2 h^{4}+\cdots$, не принадлежащую $L$ (считаем $2 \neq 0)$.

ОПРЕДЕЛЕНИЕ 3. Систему $\left\{\gamma_{i} \in L: i \in I\right\}$ формальных степенных рядов назовем $L$-суммируемой и формальный степенной ряд $\gamma$ назовем ее $L$-суммой, если, во-первых, $\gamma=\sum_{i \in I}^{\mathscr{F}} \gamma_{i}$, а во-вторых, объединение $\bigcup_{i \in I} \operatorname{supp} \gamma_{i}-$ вполне упорядоченное множество. В этом случае пишем $\gamma=\sum_{i \in I}^{L} \gamma_{i}$.

Это определение технически легче проверять с помощью следующего предложения.

ПРЕДЛОЖЕНИЕ 6. Система формальных степенных рядов $\gamma_{i} \in L(i \in I)$ L-суммируема тогда и только тогда, когда для любой $\mathbb{N}$-последовательности $i_{1}, i_{2}, i_{3}, \ldots$ попарно различных индексов $и$ любого набора әлементов $g_{j} \in \operatorname{supp} \gamma_{i_{j}}(j=1,2,3, \ldots)$ найдется пара натуральных чисел $j<k$ такая, что $g_{j}<g_{k}$. 
ДокАЗАтЕльство. С учетом предложения 1, а также того факта, что каждьй из носителей supp $\gamma_{i}$ - вполне упорядоченное множество, нам надо лишш проверить, что объединение $M=\bigcup_{i \in I} \operatorname{supp} \gamma_{i}$ является вполне упорядоченным множеством тогда и только тогда, когда для любой $\mathbb{N}$-последовательности $i_{1}, i_{2}, i_{3}, \ldots$ попарно различных индексов и любого набора элементов $g_{j} \in \operatorname{supp} \gamma_{i_{j}}(j=1,2,3, \ldots)$ найдется пара $j, k \in \mathbb{N}$ и $j<k$ такая, что $g_{j}<g_{k}$. Импликация $\Rightarrow$ очевидна. Докажем импликацию $\Leftarrow$. Предположим, что существует $\mathbb{N}$-последовательность попарно различных индексов $i_{1}, i_{2}, i_{3}, \ldots$ такая, что для некоторого набора элементов $g_{j} \in \operatorname{supp} \gamma_{i_{j}}$ имеет место бесконечная цепочка неравенств $g_{1} \geqslant g_{2} \geqslant g_{3} \geqslant \cdots$. В силу $\mathscr{F}$-суммируемости системы $\left\{\gamma_{i}\right\}$ можно считать, что все неравенства здесь строгие. Но тогда объединение $M$ не может быть вполне упорядоченным множеством.

Имеет место аналог предложения 3.

ПрЕДЛОЖЕНИЕ 7. Если $\left\{\gamma_{i}: i \in I\right\}-L$-суммируемое семейство, а $r \in F G$, $k \in K$, то семейства $\left\{r \gamma_{i}\right\},\left\{\gamma_{i} k\right\}$ также L-суммируемы $u$

$$
r \sum_{i \in I}^{L} \gamma_{i}=\sum_{i \in I}^{L} r \gamma_{i}, \quad\left(\sum_{i \in I}^{L} \gamma_{i}\right) k=\sum_{i \in I}^{L} \gamma_{i} k
$$

Доказательство следует из предложения 3.

1.2. Свертка. С помощью понятия $\mathscr{F}$-суммы операция свертки или, иначе, *-произведения $\beta * \gamma$ для $\beta=\sum_{g \in G}^{\prime} g b_{g}$ из $\mathscr{F}(G, F)$ и формальной суммы $\gamma \in$ $\mathscr{F}(G \searrow U, K)$ определяется просто и естественно:

$$
\beta * \gamma=\sum_{g \in G}^{\mathscr{F}}\left(g b_{g} \gamma\right)
$$

Разумеется, $\mathscr{F}$-сумма в правой части (6), а значит, и свертка $\beta * \gamma$ не обязаны существовать. Тем самым, свертка - частичная операция. Если $\gamma=\sum_{g \in G} g k_{g} \in$ $\mathscr{F}(G, F)$, то легко подсчитать коэффициент при $g \in G$ :

$$
(\beta * \gamma)_{g}=\sum_{g_{1}, g_{2} \in G ; g_{1} g_{2}=g} b_{g_{1}} k_{g_{2}}
$$

Для бимодуля $\mathscr{F}(G, F)$ нетрудно определить свертку любого конечного числа формальных сумм. Ограничимся случаем трех сомножителей. Пусть $\gamma \in \mathscr{F}(G \searrow U, K)$, $\beta$ - как и выше, а кроме того, дана сумма $\alpha=\sum_{g \in G}^{\prime} g a_{g}$ из $\mathscr{F}(G, F)$. Тогда по определению

$$
\alpha * \beta * \gamma=\sum_{(g, h) \in G \times G}^{\mathscr{F}}\left(g a_{g} h b_{h} \gamma\right) .
$$

Отметим без доказательства три стандартных свойства свертки.

ПРЕДЛОЖЕНИЕ 8 (ассоциативность). Предположим, что $\alpha \neq 0$ ж $\gamma \neq 0$. Если свертка $\alpha * \beta * \gamma$ определена, то *-произведения $(\alpha * \beta) * \gamma u \alpha *(\beta * \gamma)$ определены и совпадают с $\alpha * \beta * \gamma$. В частности, если определены все три *-произведения, то они совпадают. 
ПРЕДЛОЖЕНИЕ 9 (дистрибутивность). Если *-произведения $\beta * \gamma_{1} u \beta * \gamma_{2}$ для $\beta \in \mathscr{F}(G, F)$ и $\gamma_{1}, \gamma_{2} \in \mathscr{F}(G \searrow U, K)$ определень, то определена и свертка $\beta *\left(\gamma_{1}+\gamma_{2}\right)$, причем верно равенство:

$$
\beta *\left(\gamma_{1}+\gamma_{2}\right)=\beta * \gamma_{1}+\beta * \gamma_{2}
$$

Аналогично, если $\beta_{1}, \beta_{2} \in \mathscr{F}(G, F)$ u $\gamma \in \mathscr{F}(G \searrow U, K)$ таковьи, что свертки $\beta_{1} * \gamma, \beta_{2} * \gamma$ существуют, то существует свертка $\left(\beta_{1}+\beta_{2}\right) * \gamma$, причем имеет место равенство:

$$
\left(\beta_{1}+\beta_{2}\right) * \gamma=\beta_{1} * \gamma+\beta_{2} * \gamma
$$

ПРЕДЛОЖЕНИЕ 10. Групповое кольцо $F G$ является подсистемой алгебраической системь $(\mathscr{F}(G, F),+, *, 0,1)$, при этом для любого әлемента $r \in F G$ и для любых формальных сумм $\gamma \in \mathscr{F}(G \searrow U, K), \beta \in \mathscr{F}(G, F)$ имеют место равенства: $r * \gamma=r \gamma, \beta * r=\beta r$.

\section{§2. $L$-базисы}

Семейство элементов $\{g: g \in G \searrow U\}$, рассматриваемое как семейство формальных степенных рядов, обладает тем свойством, что через него в виде бесконечной линейной комбинации может быть записан любой другой ряд из $L$. В этом параграфе доказывается, что существуют и другие, естественным образом возникающие семейства формальных степенных рядов, обладающие таким же базисным свойством.

Пусть $\left\{\gamma_{i}: i \in I\right\}-$ семейство формальных степенных рядов. Скажем, что это семейство $\sigma$-суммируемо, если для любого вполне упорядоченного подмножества $M \subseteq G \searrow U$ подсемейство $\left\{\gamma_{i}: v\left(\gamma_{i}\right) \in M\right\} L$-суммируемо. Далее, назовем семейство $\left\{\gamma_{i}: i \in I\right\}$ локально полным, если для любого $h \in G \searrow U$ найдется $\gamma_{i}$ с нормой равной $h$. Это же семейство назовем монотонным, если неравенство индексов $i \neq k$ влечет неравенство норм $v\left(\gamma_{i}\right) \neq v\left(\gamma_{k}\right)$. В случае монотонности семейства можно считать, что множество индексов $I$ - подмножество линейно упорядоченного множества $G \searrow U$, причем переиндексацией можно добиться того, что большему индексу соответствует бо́льшая норма.

Семейство $\left\{\gamma_{i}: i \in I\right\}$ назовем $\sigma$-линейно независимьм, если для любого вполне упорядоченного подмножества $M \subseteq G \searrow U$ и для любого набора коэффициентов $\left\{k_{i} \in K: i \in I\right\}$ имеет место импликация:

$$
\sum_{v\left(\gamma_{i}\right) \in M}^{L} \gamma_{i} k_{i}=0 \Longrightarrow\left(\forall i \in I \Longrightarrow k_{i}=0\right)
$$

ПРЕДЛОЖЕНИЕ 11. Если $K$ - область иелостности, то монотонность влечет б-линейную независимость.

Далее до конца работы считаем, что $K$ - тело. По поводу аналога следующей теоремы см. [4] и [5]. 
ТЕорема 1. Предположим, что $\left\{\gamma_{i}: i \in I\right\}-\sigma$-суммируемое и локально полное семейство формальных степенных рядов. Тогда для любого $\gamma \in L$ найдутся коэффициенть $k_{i} \in K(i \in I)$ такие, что

$$
\gamma=\sum_{i \in I}^{L} \gamma_{i} k_{i}
$$

Если, кроме того, семейство $\left\{\gamma_{i}: i \in I\right\}$ б-линейно независимо, то коэффициенты $k_{i}$ в разложсении (7) единственны.

ДокАЗАтЕльство. Вторая часть теоремы - тривиальность. Доказываем первую часть теоремы в предположении, что $\gamma \neq 0$.

Трансфинитной индукцией по $\lambda$ будем выбирать индекс $i(\lambda) \in I$ и коэффициент $k_{\lambda}$ так, что для любых ординалов $\lambda_{1}, \lambda_{2}, \lambda_{1}<\lambda_{2} \leqslant \lambda$, имеют место соотношения:

$$
\begin{gathered}
v\left(\gamma_{i\left(\lambda_{1}\right)}\right)<v\left(\gamma_{i\left(\lambda_{2}\right)}\right) \\
\left\{\gamma_{i(\mu)}: \mu<\lambda_{2}\right\}-L \text {-суммируемо и } v\left(\gamma-\sum_{\mu<\lambda_{2}}^{L} \gamma_{i(\mu)} k_{\mu}\right)=v\left(\gamma_{i\left(\lambda_{2}\right)}\right)
\end{gathered}
$$

Основание индукции $(\lambda=1)$. Пользуясь полнотой, для нормы $v(\gamma)$ найдем индекс $i(1)$ такой, что $v\left(\gamma_{i(1)}\right)=v(\gamma)$. Тогда $\partial\left(\gamma_{i(1)} k_{1}\right)=\partial \gamma$ для подходяшего $k_{1} \in K$, учитывая, что $K-$ тело.

Индукционный переход. Предположим, что для всех ординалов $\mu$, меньших $\lambda$, индексы $i(\mu)$ и коэффициенты $k_{\mu}$, удовлетворяюшие (8) и (9) для $\lambda_{1}<\lambda_{2} \leqslant \mu$, выбраны.

Прежде всего убедимся, что семейство $\left\{\gamma_{i(\mu)}: \mu<\lambda\right\} L$-суммируемо. Если $\lambda$ - непредельньй ординал, то это следствие общего факта: если к $L$-суммируемому семейству присовокупить конечное множество формальных степенных рядов, то получим снова $L$-суммируемое семейство. Если же $\lambda$ - предельный ординал, то (8) верно для любых $\lambda_{1}<\lambda_{2}$, поэтому $L$-суммируемость семейства $\left\{\gamma_{i(\mu)}: \mu<\lambda\right\}$ вытекает из $\sigma$-суммируемости семейства $\left\{\gamma_{i}\right\}$.

Если $\gamma=\sum_{\mu<\lambda}^{L} \gamma_{i(\mu)} k_{\mu}$, то первое утверждение теоремы доказано и индукционное построение завершено. Предположим, что

$$
\beta=\gamma-\sum_{\mu<\lambda}^{L} \gamma_{i(\mu)} k_{\mu} \neq 0
$$

Тогда, как и вьше, находим индекс $i(\lambda) \in I$ и элемент $k_{\lambda} \in K$ с условием

$$
\partial\left(\gamma_{i(\lambda)} k_{\lambda}\right)=\partial \beta
$$

Следовательно, равенство (9) для $\lambda_{2}=\lambda$, а значит, и для всех $\lambda_{2} \leqslant \lambda$ имеет место. Остается проверить неравенство (8) лиш в частном случае $\lambda_{2}=\lambda$.

Случай 1. $\lambda$-предельный ординал. Обозначим $\tau=\lambda_{1}+1$ и заметим, что этот ординал также меньше $\lambda$. Имеем:

$$
\beta=\gamma-\sum_{\mu<\lambda}^{L} \gamma_{i(\mu)} k_{\mu}=\left(\gamma-\sum_{\mu<\tau}^{L} \gamma_{i(\mu)} k_{\mu}\right)-\sum_{\tau \leqslant \mu<\lambda}^{L} \gamma_{i(\mu)} k_{\mu}
$$


Разность, стоящая в скобках в правой части (10), имеет норму равную $v\left(\gamma_{i(\tau)}\right)$ (см. $(9))$, что строго больше чем $v\left(\gamma_{i\left(\lambda_{1}\right)}\right)$. Каждое из слагаемых во второй сумме в (10) также имеет норму большую чем $v\left(\gamma_{i\left(\lambda_{1}\right)}\right)$ (опять используем (8)). Следовательно, $v\left(\gamma_{i(\lambda)}\right)=v(\beta)>v\left(\gamma_{i\left(\lambda_{1}\right)}\right)$.

Случай 2. $\lambda=\tau+1-$ непредельный ординал. Тогда

$$
v\left(\gamma_{i(\lambda)}\right)=v(\beta)=v\left(\gamma-\sum_{\mu<\tau}^{L} \gamma_{i(\mu)} k_{\mu}-\gamma_{i(\tau)} k_{\tau}\right)>v\left(\gamma_{i(\tau)} k_{\tau}\right)=v\left(\gamma_{i(\tau)}\right)
$$

ибо

$$
\partial\left(\gamma-\sum_{\mu<\tau}^{L} \gamma_{i(\mu)} k_{\mu}\right)=\partial \gamma_{i(\tau)} k_{\tau}
$$

и, значит, разность этих двух формальных степенных рядов имеет бо́льшую норму. Итак, мы проверили (8).

Продолжая индукционный процесс, рано или поздно мы придем к ординалу $\lambda$ такому, что $\gamma=\sum_{\mu<\lambda}^{L} \gamma_{i(\mu)} k_{\mu}$. Это следует из ограниченности сверху порядковых типов вполне упорядоченных подмножеств любого фиксированного линейно упорядоченного множества.

Теорема доказана.

ОПредЕЛЕНиЕ 4. Систему формальных степенных рядов $\left\{\gamma_{h}: h \in G \searrow U\right\}$ назовем $L$-базисом, если она монотонна, полна и $\sigma$-суммируема.

Без ограничения общности можно считать, что для $L$-базиса неравенство $h_{1}<h_{2}$ влечет неравенство $v\left(\gamma_{h_{1}}\right)<v\left(\gamma_{h_{2}}\right)$. Такого рода условие монотонности и будет предполагаться в дальнейшем. Пример $L$-базисов доставляет следующее предложение.

ПРЕДЛОЖЕНИЕ 12. Для любого ненулевого элемента $r \in F G$ система $\{r \cdot h: h \in G \searrow U\}$ будет L-базисом.

ДоказАтЕльство. Предполагаем, что $r$ имеет вид (4). Для любого $g \in G$ справедливо равенство:

$$
v(r \cdot g)=\min \left\{\overline{g_{1} g}, \ldots, \overline{g_{k} g}\right\}
$$

Докажем полноту семейства $\{r h\}$. Пусть элемент $h \in G \searrow U$ произволен. Выберем в множестве $\left\{g_{1}^{-1} h, \ldots, g_{k}^{-1} h\right\}$ максимальный элемент. Без ограничения общности можно предполагать, что этим элементом будет $g=g_{1}^{-1} h$. Утверждается, что $\bar{g}$ - искомый элемент, т.е. $v(r \cdot \bar{g})=h$. Действительно, $v(r \cdot \bar{g})=v(r \cdot g)(\mathrm{cm.}$ лемму 1, г)). Но $g_{i} g \geqslant g_{1} g=h$, ибо $g=g_{1}^{-1} h \geqslant g_{i}^{-1} h$ для любого $i$. Отсюда вытекает полнота.

Условие $\sigma$-суммируемости следует из предложения 7.

Докажем теперь монотонность. Пусть $h_{1}<h_{2}$ - элементы из $G \searrow U$ и $v\left(r \cdot h_{1}\right)=\bar{g}_{i} h_{1}$, a $v\left(r \cdot h_{2}\right)=\bar{g}_{j} h_{2}$. Тогда

$$
{\overline{g_{i}}}_{1} \leqslant{\overline{g_{j}}}_{1}<{\overline{g_{j}}}_{2}
$$

в силу (11) и того, что порядок “<” выдерживает умножение слева (лемма 1, в)). 
Заметим, что формальньй степенной ряд (1) мы можем записать и так: $\gamma=$ $\sum_{h \in G \searrow U}^{L} h k_{h}$. То же самое верно для любого $L$-базиса $\left\{\gamma_{h}: h \in G \searrow U\right\}$. А именно в силу теоремы 1 получаем, что тот же степенной ряд $\gamma$ может быть единственным образом представлен в виде $\gamma=\sum_{h \in G \searrow U}^{L} \gamma_{h} \widetilde{k}_{h}\left(\widetilde{k}_{h} \in K\right)$. Конечно же, понятие $L$-базиса связано с автоморфизмами линейного пространства $L_{K}$, обладающими соответствующими свойствами.

ОПРЕДЕЛЕНИЕ 5. Линейный оператор $q: L_{K} \rightarrow L_{K}$ назовем локально монотонным, если для любых $g, h \in G \searrow U$ неравенство $g<h$ влечет неравенство норм: $v(q[g])<v(q[h])$. Этот же оператор назовем $\sigma$-линейным, если образы $q\left[\beta_{i}\right], i \in I$, любой $L$-суммируемой системы формальных степенных рядов $\left\{\beta_{i} \in L: i \in I\right\}$ составляют также $L$-суммируемую систему, причем

$$
q\left[\sum_{i \in I}^{L} \beta_{i}\right]=\sum_{i \in I}^{L} q\left[\beta_{i}\right] .
$$

Эндоморфизм $q$ назовем локально сюрвективным, если для любого $h \in G \searrow U$ найдется элемент $g \in G \searrow U$ такой, что $v(q[g])=h$.

Если $q$ одновременно локально монотонен, локально сюръективен и $\sigma$-линеен,

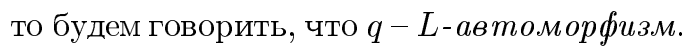

Сформулируем основной результат работы.

Teopema 2.

1. Всякий $L$-автоморфизм является автоморфизмом пространства $L_{K}$

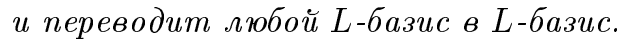

2. Обратное отображсение $\kappa L$-автоморфизму такжсе будет L-автоморфизмом. Более того, множсество всех L-автоморфизмов составляет подаруппу в группе автоморфизмов Aut $L_{K}$.

3. Eсли $\left\{\beta_{h}: h \in G \searrow U\right\} u\left\{\gamma_{h}: h \in G \searrow U\right\}-\partial в а L-б а з и с а$, то отображение $q: L \rightarrow L$, заданное формулой

$$
\sum_{h \in G \searrow U}^{L} \beta_{h} k_{h} \rightarrow \sum_{h \in G \searrow U}^{L} \gamma_{h} k_{h}
$$

будет L-автоморфизмом.

Сначала докажем две леммы.

Лемма 2. Пусть отображсние $q: L \rightarrow L$ таково, что для любого вполне упорядоченного подмножества $M \subseteq G \searrow U$ система формальных степенных рядов $\{q[h]: h \in M\} L$-суммируема и выполняется равенство

$$
q\left[\sum_{h \in M}^{\prime} h k_{h}\right]=\sum_{h \in M}^{L} q[h] k_{h}
$$

для любого набора коэффициентов $k_{h} \in K$. Тогда $q-\sigma$-линейньй эндомор$\oint и з м$. 
ДокаЗАТЕльство. Пусть $\left\{\gamma_{i} \in L: i \in I\right\}-L$-суммируемое семейство. Тогда $N=\bigcup_{i \in I} \operatorname{supp} \gamma_{i}$ будет вполне упорядоченным множеством. Из условия вытекает, что и $M=\bigcup_{h \in N} \operatorname{supp} q[h]$ также будет вполне упорядоченньпм множеством. Пусть

$$
\gamma_{i}=\sum_{h \in N}^{\prime} h k_{h}^{i} \quad(i \in I), \quad q[h]=\sum_{g \in M}^{\prime} g k_{g}^{h} \quad(h \in N)
$$

Тогда

$$
q\left[\gamma_{i}\right]=\sum_{h \in N}^{L} q[h] k_{h}^{i}=\sum_{g \in M}^{\prime} g\left(\sum_{h \in N} k_{g}^{h} k_{h}^{i}\right)
$$

по определению $L$-суммы. Далее,

$$
\begin{gathered}
\sum_{i \in I}^{L} \gamma_{i}=\sum_{h \in N}^{\prime} h\left(\sum_{i \in I} k_{h}^{i}\right) \\
q\left[\sum_{i \in I}^{L} \gamma_{i}\right]=\sum_{h \in N}^{L} q[h]\left(\sum_{i \in I} k_{h}^{i}\right)=\sum_{g \in M}^{\prime} g\left(\sum_{h \in N} k_{g}^{h} \sum_{i \in I} k_{h}^{i}\right)
\end{gathered}
$$

Для фиксированного $g \in M$ почти все произведения $k_{g}^{h} k_{h}^{i}$ равны нулю. Действительно, найдется лишь конечное число $h \in N$ таких, что $k_{g}^{h} \neq 0$; это следует из $L$-суммируемости $\{q[h]: h \in N\}$. Для каждого из таких $h$ найдется лишь конечное число индексов $i$ с условием $k_{h}^{i} \neq 0$ - это следствие $L$-суммируемости $\left\{\gamma_{i}\right\}$. Тогда

$$
\sum_{h \in N, i \in I} k_{g}^{h} k_{h}^{i}=\sum_{h \in N} k_{g}^{h} \sum_{i \in I} k_{h}^{i}=\sum_{i \in I}\left(\sum_{h \in N} k_{g}^{h} k_{h}^{i}\right) .
$$

Кроме того, $\bigcup_{i \in I} \operatorname{supp} q\left[\gamma_{i}\right]$ - подмножество вполне упорядоченного множества $M$, а значит, оно само вполне упорядочено. Следовательно, система $\left\{q\left[\gamma_{i}\right]\right\} L$-суммируема и

$$
\sum_{i \in I}^{L} q\left[\gamma_{i}\right]=\sum_{g \in M}^{\prime} g\left(\sum_{i \in I}\left(\sum_{h \in N} k_{g}^{h} k_{h}^{i}\right)\right)=\sum_{g \in M}^{\prime} g\left(\sum_{h \in N} k_{g}^{h} \sum_{i \in I} k_{h}^{i}\right)=q\left[\sum_{i \in I}^{L} \gamma_{i}\right]
$$

Из определения отображения $q$ вытекает сразу его однородность: $q[\gamma k]=q[\gamma] k$ для любых $\gamma \in L, k \in K$.

Тем самьм, лемма полностью доказана.

Обозначим через $\widehat{\Gamma}$ дедекиндово замькание линейно упорядоченного множества $G \searrow U$. В множестве $\widehat{\Gamma} \cup\{\infty\}$ любое подмножество имеет точную верхнюю грань. Если $\gamma \in L, \mathbf{a} \varepsilon \in \widehat{\Gamma} \cup\{\infty\}$, то обозначим через $(\gamma)_{<\varepsilon} \varepsilon$-начало ряда $\gamma$, т.е. такой формальный степенной ряд $\beta$, что $\operatorname{supp} \beta<\varepsilon$ и $v(\gamma-\beta) \geqslant \varepsilon$. Если положить

$$
\gamma=(\gamma)_{<\varepsilon}+\delta
$$

то $\operatorname{supp}(\gamma)_{<\varepsilon}<\varepsilon \leqslant \operatorname{supp} \delta$. Разложение (13) назовем $\varepsilon$-сечением ряда $\gamma$.

ЛЕмма 3. Если $q$-монотонный и б-линейный автоморфизм, то $q^{-1}$ также $\sigma$-линеен. 
ДокАЗАТЕЛЬСтво. Предположим, что $q^{-1}$ не является $\sigma$-линейным эндоморфизмом. Тогда согласно лемме 2 найдется вполне упорядоченное подмножество $M \subseteq G \searrow U$ такое, что система $\left\{\gamma_{h}=q^{-1}[h]: h \in M\right\}$ не $L$-суммируема. Действительно, если бы система $\left\{\gamma_{h}=q^{-1}[h]: h \in M\right\}$ была $L$-суммируема, то равенство $q^{-1}\left[\sum_{h \in M}^{L} \gamma_{h}\right]=\sum_{h \in M}^{L} q^{-1}\left[\gamma_{h}\right]$ выполнялось бы автоматически, ибо оно эквивалентно равенству

$$
\sum_{h \in M}^{L} \gamma_{h}=q\left[\sum_{h \in M}^{L} q^{-1}\left[\gamma_{h}\right]\right]
$$

справедливому в силу $\sigma$-линейности $q$.

Обозначим

$$
\nu=\sup \left\{\varepsilon \in \widehat{\Gamma} \cup\{\infty\}: \text { система }\left\{\left(\gamma_{h}\right)_{<\varepsilon}: h \in M\right\} L \text {-суммируема }\right\} \text {. }
$$

Так как $\left((\gamma)_{<\varepsilon}\right)_{<\rho}=(\gamma)_{<\rho}$ для любых $\varepsilon, \rho \in \widehat{\Gamma} \cup\{\infty\}, \rho \leqslant \varepsilon$, то нетрудно видеть, что для любого $\rho \in \widehat{\Gamma} \cup\{\infty\}$, строго меньшего чем $\nu$, система $\left\{\left(\gamma_{h}\right)_{<\rho}: h \in M\right\}$ будет суммируемой.

Более того, утверждаем, что система $\left\{\left(\gamma_{h}\right)_{<\nu}: h \in M\right\} L$-суммируема. Действительно, пусть $h_{1}<h_{2}<h_{3}<\cdots-\mathbb{N}$-последовательность элементов из $M$ и элементы $z_{i} \in \operatorname{supp}\left(\gamma_{h_{i}}\right)_{<\nu}(i \in \mathbb{N})$ выбраны произвольно. Допустим, что элемент $z=z_{1}$ строго больше всех остальных $z_{i}, i>1$. Так как $z \in \operatorname{supp}\left(\gamma_{h_{1}}\right)_{<\nu}$, то $z<\nu$. Следовательно, система $\left\{\left(\gamma_{h}\right)_{<z}: h \in M\right\} L$-суммируема. Но $z_{i} \in \operatorname{supp}\left(\gamma_{h_{i}}\right)_{<z}$ для $i>1$, ибо $z_{i}<z$. Тогда $z_{i}<z_{j}$ для какой-либо пары натуральных чисел $i<j$ (см. предложение 6). Если же $z$ не является максимальным элементом, то $z_{1}<z_{i}$ для подходящего $i \in \mathbb{N}$. Мы показали, что выполнены условия предложения 6 ; отсюда следует, что система $\left\{\left(\gamma_{h}\right)_{<\nu}: h \in M\right\} L$-суммируема.

Обозначим $\alpha_{h}=\left(\gamma_{h}\right)_{<\nu}$, и пусть $\gamma_{h}=\alpha_{h}+\beta_{h}$ будет $\nu$-сечением. Так как система $\left\{\gamma_{h}\right\}$ не является $L$-суммируемой по предположению, а система $\left\{\alpha_{h}\right\} L$-суммируема по доказанному выше, то система $\left\{\beta_{h}\right\}$ не будет $L$-суммируемой. Более того, множество норм $\left\{v\left(\beta_{h}\right): h \in M\right\}$ не будет вполне упорядоченньг, иначе нашелся бы элемент $g \in \bigcup_{h \in M} \operatorname{supp} \beta_{h}$, а он заведомо больше $\nu$, такой, что система $\left\{\left(\beta_{h}\right)_{<g}: h \in M\right\}$, а значит, и система $\left\{\left(\gamma_{h}\right)_{<g}=\alpha_{h}+\left(\beta_{h}\right)_{<g}: h \in M\right\}$ $L$-суммируемы, что противоречит максимальности элемента $\nu$.

Итак, множество $\left\{v\left(\beta_{h}\right): h \in M\right\}$, а значит, и множество $\left\{v\left(q\left[\beta_{h}\right]\right): h \in M\right\}$ не являются вполне упорядоченными (пользуемся монотонностью автоморфизма $q$ ). С другой стороны,

$$
q\left[\beta_{h}\right]=q\left[\gamma_{h}\right]-q\left[\alpha_{h}\right]=h-q\left[\alpha_{h}\right]
$$

где системы $\{h: h \in M\}$ и $\left\{q\left[\alpha_{h}\right]: h \in M\right\}$ будут $L$-суммируемы, причем вторая система $L$-суммируема в силу $\sigma$-линейности автоморфизма $q$. Значит, и их разность должна быть $L$-суммируемой. Полученное противоречие показьвает, что наше первоначальное допушение было неверным. Следовательно, автоморфизм $q^{-1} \sigma$-линеен. 
ДокаЗАТЕЛЬСТво теоРемы 2. 1. Пусть $q-L$-автоморфизм. Тогда система формальных степенных рядов $\{q[h]: h \in G \backslash U\}$ будет $L$-базисом, ибо монотонность и полнота этой системы есть в точности локальная монотонность и локальная сюръективность эндоморфизма $q$, а $\sigma$-суммируемость системы вытекает из $\sigma$-линейности $q$, если положить в определении $5 I=G \backslash U$ и $\beta_{g}=g$. Более того,

$$
q\left[\sum_{h \in G \backslash U}^{\prime} h k_{h}\right]=\sum_{h \in G \backslash U}^{L} q[h] k_{h}
$$

для любого формального степенного ряда $\gamma=\sum_{h \in G \backslash U}^{L} h k_{h}$. Из (14) получаем мономорфность $q$, а именно из равенства $q[\gamma]=0$ вытекает, что $k_{h}=0$ для всех $h \in G \searrow U$. Эпиморфность $q$ получается также из (14), если применить теорему 1 к системе формальных степенных рядов $\gamma_{h}=q[h](h \in G \searrow U)$.

Далее, пусть $\left\{\beta_{h}: h \in G \backslash U\right\}-L$-базис. Тогда система $\left\{q\left[\beta_{h}\right]: h \in G \backslash U\right\}$ $\sigma$-суммируема, ибо эндоморфизм $q$ по условию $\sigma$-линеен. Монотонность и полнота этой системы следуют из локальной монотонности и локальной сюръективности $q$, если учесть равенство

$$
v(q[\gamma])=v(q[v(\gamma)]),
$$

верное для любого формального степенного ряда $\gamma$ и для любого локально монотонного и $\sigma$-линейного эндоморфизма. Докажем это утверждение. Можно считать $\gamma \neq 0$. Тогда $\gamma=\partial \gamma+\delta$, где $\operatorname{supp} \delta>\operatorname{supp}(\partial \gamma)=\{v(\gamma)\}$. Из локальной монотонности $q$ вытекает, что $v(q[g])>v(q[\partial \gamma])$ для любого $g \in \operatorname{supp} \delta$. Тогда $\sigma$-линейность эндоморфизма $q$ дает неравенство $v(q[\delta])>v(q[\partial \gamma])$. Так как $q[\gamma]=q[\partial \gamma]+q[\delta]$, то получаем

$$
v(q[\gamma])=v(q[\partial \gamma])=v(q[v(\gamma)]),
$$

ибо $\partial \gamma=v(\gamma) k$ для некоторого ненулевого $k \in K$.

2. Пусть $q-L$-автоморфизм. Тогда $q^{-1}$ локально монотонен, так как неравенство $v\left(q^{-1}[g]\right) \geqslant v\left(q^{-1}[h]\right)$ для $g, h \in G \backslash U$ влечет $g \geqslant h$ в силу (15). По лемме 3 автоморфизм $q^{-1} \sigma$-линеен. Далее, $q^{-1}$ локально сюръективен. Действительно, для любого $h \in G \backslash U$ верно равенство

$$
h=v\left(q^{-1}[q[h]]\right)=v\left(q^{-1}[v(q[h])]\right)
$$

(см. (15); необходимо учесть также, что $q^{-1} \sigma$-линеен и локально монотонен). Значит, $g=v(q[h])$ - искомый элемент из $G \searrow U$, т.е. $v\left(q^{-1}[g]\right)=h$.

Осталось показать, что композиция двух $L$-автоморфизмов будет снова $L$-автоморфизмом. Из первого утверждения теоремы вытекает, что эта композиция будет переводить стандартный $L$-базис $G \searrow U$ в $L$-базис. По лемме 2 композиция будет $\sigma$-линейной. Локальная монотонность и локальная сюръективность снова легко получаются с применением равенств типа (15). Следовательно, композиция будет $L$-автоморфизмом.

3. Разложим отображение (12) в композицию двух: $q=s \circ p^{-1}$, где

$$
p\left[\sum_{g \in G \backslash U}^{\prime} g k_{g}\right]=\sum_{g \in G \backslash U}^{L} \beta_{g} k_{g} ; \quad s\left[\sum_{g \in G \backslash U}^{\prime} g k_{g}\right]=\sum_{g \in G \backslash U}^{L} \gamma_{g} k_{g} .
$$


Каждое из $p$ и $s$ будет $L$-автоморфизмом в силу леммы 2 , поэтому остается применить второе утверждение теоремы 2 .

Доказательство теоремы 2 завершено.

СлеДСТВИЕ (см. [4]). Пусть $r \in F G$ - ненулевой элемент. Тогда умножение слева на әлемент $r$ - L-автоморфизм и система $\left\{r^{-1}[g]: g \in G \searrow U\right\}$, состоящая из формальных степенных рядов $r^{-1}[g]$ таких, что $r \cdot r^{-1}[g]=g$, будет L-базисом.

Более того, для любого формального степенного ряда $\gamma$ вида (1) определен формальный степенной ряд

$$
r^{-1}[\gamma]=\sum_{h \in G}^{L} r^{-1}[h] k_{h}
$$

являющийся единственным решением уравнения $r \cdot X=\gamma$.

ДокАЗАТЕЛьство. Согласно предложению 12 система $\{r \cdot h: h \in G \searrow U\}$ будет $L$-базисом. Остается применить теорему 2.

\section{§3. Случай чистого конуса}

В этом параграфе предполагается, что $P$ - чистый конус в группе $G$ и тем самьгм $K=F$; как и ранее, $L-(F G-F)$-бимодуль формальных степенных рядов, а $R-(F-F G)$-бимодуль формальных степенных рядов относительно правого порядка " $\leqslant r "($ см. $(2))$.

Теорема 3. Для любих формальных степенных рядов $\gamma \in L u \beta \in R$ свертка $\gamma * \beta$ определена. Кроме того, если носитель $\operatorname{supp} \beta$ лежст в нормализаmоре $N(P)$ конуса $P$, то $\gamma * \beta \in L$.

ДокАЗАтЕльство. Перенумеруем элементы носителя $\operatorname{supp} \beta$ в возрастаюшем поря дке относительно отношения " $\leqslant r "$ :

$$
\operatorname{supp} \beta=\left\{h_{1}, h_{2}, h_{3}, \ldots\right\}, \quad h_{1}<_{r} h_{2}<_{r} h_{3}<_{r} \cdots
$$

Пусть элемент $g \in G$ произволен. Тогда

$$
(\gamma * \beta)_{g}=\sum_{i}(\gamma)_{g h_{i}^{-1}}(\beta)_{h_{i}}
$$

Неравенство $h_{i}<_{r} h_{i+1}$ дает $h_{i+1} h_{i}^{-1} \in P$, следовательно, $h_{i}^{-1}>h_{i+1}^{-1}$, значит, $g h_{i}^{-1}>g h_{i+1}^{-1}$. Так как $\gamma \in L$, то пересечение множества $\left\{g h_{i}^{-1}\right\}$ с носителем supp $\gamma$ конечно, откуда вытекает, что сумма (16) конечна и определена.

Теперь предположим, что $h_{i} P=P h_{i}$ для любого ординала $i$. Пусть $g_{1}>g_{2}>$ $g_{3}>\cdots-$ бесконечная цепочка элементов из групшы $G$. Легко доказывается 
Лемма 4. Если $a \geqslant b$ в $G$ u $h P=P h$, mo $a h \geqslant b h$.

Пользуясь этой леммой, получаем:

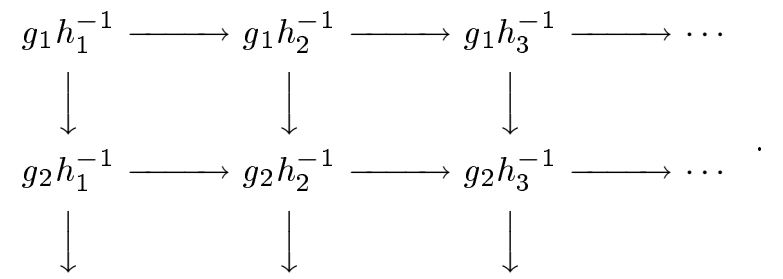

Здесь стрелки заменяют знак неравенства ">". Из системы неравенств (17) вытекает, что пересечение $\left\{g_{j} h_{i}^{-1}\right\} \cap \operatorname{supp} \gamma$ конечно. Следовательно, $\operatorname{supp}(\gamma * \beta)$ не может содержать бесконечно убывающей цепочки элементов по отношению к "<".

ЗАмечАнИЕ. Если $\gamma=1-m \in L, \operatorname{supp} m>1$ и все элементы из $\operatorname{supp} m$ лежат в нормализаторе $N(P)$, то набор степеней $\left\{m^{i}: i=0,1,2, \ldots\right\}$ будет как $L$-суммируемой, так и $R$-суммируемой системой, и сумма $\delta=1+m+m^{2}+m^{3}+\cdots$, принадлежашая $L \cap R$, удовлетворяет соотношению $\gamma \delta=\delta \gamma=1$. Это достаточно просто следует из результата А.И. Мальцева [1]. Действительно, обозначим через $G_{0}$ подгруппу группы $G$, порожденную элементами из носителя $\operatorname{supp} m$. Тогда $P_{0}=P \cap G_{0}$ будет чистьм инвариантным конусом в $G_{0}$. Тем самым, он будет задавать линейный порядок, и примени́м результат Мальцева, утверждающий, что в этом случае $L_{0}=R_{0}$ - тело относительно свертки и покомпонентного сложения.

В следуюшей лемме фигурирует оператор $\partial_{r}$. Он определяется точно так же, как и $\partial$, но относительно правого порядка " $\leqslant r$ ".

Лемма 5. Пусть $\gamma \in L, \beta \in R ; \partial \gamma=a h, \partial_{r}(\beta)=k g ; a, k \in K, h, g \in G$. Тогда

$$
(\gamma * \beta)_{h g}=a k h g
$$

В частности, $\gamma * \beta \neq 0$. Кроме того, если $g=1$, то $\partial(\gamma * \beta)=(a k) h$.

ДокаЗАтельство. Пусть $g^{\prime} \in \operatorname{supp} \beta, h^{\prime} \in \operatorname{supp} \gamma$. Тогда $g^{\prime} \geqslant_{r} g$ и $h^{\prime} \geqslant h$. Следовательно, $g^{\prime} g^{-1}, h^{-1} h^{\prime} \in P$. Если $g^{\prime} \neq g$ или $h^{\prime} \neq h$, то $h^{-1} h^{\prime} g^{\prime} g^{-1}>1$ и, значит, $h^{\prime} g^{\prime} \neq h g$. Отсюда получаем равенство $(\gamma * \beta)_{h g}=(a k) h g$.

Предположим, что $g=1$, а $g^{\prime} \neq 1$ - как и выше. Тогда $g^{\prime}>1$, откуда $h^{\prime} g^{\prime}>$ $h^{\prime} \geqslant h$ и последнее утверждение леммы доказано.

Теперь ответим на вопрос: когда $*$-произведение не равно нулю?

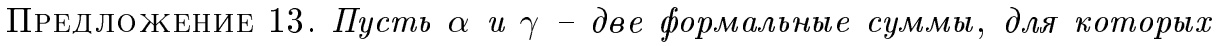
существует *-произведение $\alpha * \gamma$. Предположим, что по крайней мере одно из следующих предполохсений верно:

1) существуют норми $g=v(\gamma) u v(\alpha \cdot g)$;

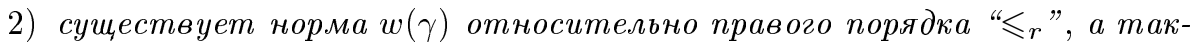
же существует $v(\alpha)$.

Тогда $\alpha * \gamma \neq 0$. Более того, в первом случае имеет место равенство $v(\alpha * \gamma)=v(\alpha g)$. 
ДокАЗАТЕльство. Предположим, что верно первое предположение. Тогда $\gamma=g\left(a+\delta_{1}\right)$, где $a \in K^{*}$ и $\operatorname{supp} \delta>1$. Аналогично, $\alpha g=h\left(b+\delta_{2}\right)$, где $b \in K^{*}$, $h=v(\alpha g)$ и $\operatorname{supp} \delta_{2}>1$. Тогда

$$
\alpha * \gamma=(\alpha g) *\left(a+\delta_{1}\right)=h\left(b+\delta_{2}\right) *\left(a+\delta_{1}\right)=h\left(b a+\delta_{2} a+b \delta_{1}+\delta_{2} * \delta_{1}\right) .
$$

Так как носители $\operatorname{supp}\left(\delta_{2} a\right), \operatorname{supp}\left(b \delta_{1}\right), \operatorname{supp}\left(\delta_{2} * \delta_{1}\right)$ больше единичного элемента $1_{G}$ и $b a \neq 0$, то $\alpha * \gamma \neq 0$.

В случае, когда верно второе предположение, достаточно применить лемму 5.

СледСТВИЕ. Если $\gamma \in L u \beta \in R$ - ненулевые степенные ряды, то свертка $\gamma * \beta$ существует и также не равна нулю.

ПримеР. Приведем пример уравнения $X r=1$ с ненулевым $r \in F G$, которое не имеет решений в $L$. Пусть $G=\left\{x, y: x y^{2} x=y\right\}-$ группа трилистника, где подмоноид $P=\operatorname{semigr}\{x, y\}$ является чистьм конусом (см. [6]). Возьмем $r=1-x+y$. Предположим, что $\gamma \in L-$ решение уравнения

$$
X(1-x+y)=1
$$

Тогда $1=\partial(\gamma(1-x-y))=\partial \gamma$ по лемме 5 . Так как подполугруппа $J=$ $\operatorname{semigr}\left\{x^{n} y: n \in \mathbb{Z}\right\}$ - двусторонний идеал в конусе $P$, а $P \backslash J=\left\{1, x, x^{2}, \ldots, x^{n}, \ldots\right\}$, получаем $\gamma_{J}(1-x)=1$, где $\gamma_{J}-$ начало ряда $\gamma$, содержашее все слагаемые вида $k x^{i}(k \in F)$. Отсюда легко получаем $\gamma_{J}=1+x+x^{2}+x^{3}+\cdots$. Кроме того, $\gamma=\gamma_{J}+\delta$, где $v(\delta) \in J$. Но

$1=\gamma(1-x+y)=\left(\gamma_{J}+\delta\right)(1-x+y)=1+y+x y+x^{2} y+x^{3} y+\cdots+\delta-\delta x+\delta y$

Отсюда: $y+x y+x^{2} y+x^{3} y+\cdots=\delta x-\delta y-\delta$. Для любого натурального $n$ элемент $x^{n} y \notin \operatorname{supp}(\delta \cdot y)$, иначе $x^{n} \in \operatorname{supp} \delta$, что невозможно в силу $v(\delta) \in J$. Таким образом, $\delta y=0$ и $\delta=0$, откуда $y+x y+x^{2} y+\cdots=0$ - противоречие. Противоречие показывает, что уравнение (18) не имеет решения в $L$.

Что же касается решения уравнения $(1-x+y) X=1$, то оно выглядит так:

$$
X=\sum_{n=0}^{\infty} y^{3 n}(1-x)^{-1}\left(x(1-y x)^{-1}(1-x)^{-1}\right)^{n+1}\left[1_{\mathscr{F}}\right]
$$

Заметим, что $(1-k h)^{-1}[g]$ для $h, g \in G ; k \in F^{*}$ имеет вид либо $g+k h g+$ $k^{2} h^{2} g+\cdots$ в случае $g<h g$, либо $-k^{-1} h^{-1} g-k^{-2} h^{-2} g-\cdots$ в случае $g>h g$.

Лемма 6. Пусть әлемент $r \in F G$ записан в виде (4), а әлемент $h$ взят из носителя какого-либо формального степенного ряда $\gamma$. Выберем индекс $i_{*}$, для которого $g_{i_{*}} h=\min \left\{g_{1} h, \ldots, g_{m} h\right\}$, и предположим, что $g_{i_{*}} h \notin \operatorname{supp}(r \gamma)$. Тогда найдется әлемент $h^{\prime} \in \operatorname{supp} \gamma$ такой, что $h^{\prime}<h u g_{j} h^{\prime}=g_{i_{*}} h$ для некоторого $j$. 
ДокАЗАТЕЛЬСтво. Без ограничения общности можно предположить, что $i_{*}=1$. Так как $r \gamma=a_{1} g_{1} \gamma+a_{2} g_{2} \gamma+\cdots+a_{m} g_{m} \gamma$ и $g_{1} h \in \operatorname{supp}\left(g_{1} \gamma\right)$, но $g_{1} h \notin \operatorname{supp}(r \gamma)$, то $g_{1} h \in \operatorname{supp}\left(a_{j} g_{j} \gamma\right)$ для некоторого $j \geqslant 2$. В этом случае $g_{1} h=g_{j} h^{\prime}$ для подходящего $h^{\prime} \in \operatorname{supp} \gamma$. В силу того что $g_{1} h<g_{j} h$ согласно выбору $g_{1}$, получаем: $g_{j} h>g_{j} h^{\prime}$. Таким образом, $h>h^{\prime}$, что и требовалось доказать.

ТЕОРема 4. Пусть әлемент $r \in F G$ записан в виде (4), а $\gamma \in L$-решение уравнения $r X=1$. Тогда каждый элемент $h \in \operatorname{supp} \gamma$ имеет вид:

$$
h=g_{i_{1}}^{-1} g_{j_{1}} g_{i_{2}}^{-1} g_{j_{2}} \cdots g_{i_{k}}^{-1} g_{j_{k}} g_{j}^{-1}
$$

$2 \partial e$

$$
g_{j}^{-1}<g_{i_{k}}^{-1} g_{j_{k}} g_{j}^{-1}<g_{i_{k-1}}^{-1} g_{j_{k-1}} g_{i_{k}}^{-1} g_{j_{k}} g_{j}^{-1}<\cdots<h,
$$

причем все әлементы әтой чепочки принадлежсат носителю supp $\gamma$.

ДокАЗАТЕЛьство. Пусть $g_{i_{1}} h=\min _{1 \leqslant i \leqslant m}\left\{g_{i} h\right\}$. Если $g_{i_{1}} h=1$, то $j=i_{1}$, $h=g_{j}^{-1}$ и доказательство завершено. В противном случае $g_{i_{1}} h \notin \operatorname{supp}(r \gamma)$. Поэтому, используя лемму 6 , можно найти элемент $h^{\prime} \in \operatorname{supp} \gamma$, для которого $h^{\prime}<h$ и $g_{j_{1}} h^{\prime}=g_{i_{1}} h$ при некотором $j_{1}\left(1 \leqslant j_{1} \leqslant m\right)$. Это дает: $h=g_{i_{1}}^{-1} g_{j_{1}} h^{\prime}$. Далее применяем трансфинитную индукцию по вполне упорядоченному множеству supp $\gamma$ и записываем $h^{\prime}$ в виде $h^{\prime}=g_{i_{2}}^{-1} g_{j_{2}} \cdots g_{i_{k}}^{-1} g_{j_{k}} g_{j}^{-1}$, причем

$$
g_{j}^{-1}<g_{i_{k}}^{-1} g i_{k} g_{j}^{-1}<g_{i_{k-1}}^{-1} g_{j_{k-1}} g_{i_{k}}^{-1} g_{j_{k}} g_{j}^{-1}<\cdots<h^{\prime}=g_{i_{2}}^{-1} g_{j_{2}} \cdots g_{i_{k}}^{-1} g_{j_{k}} g_{j}^{-1}
$$

и каждое звено этой цепочки принадлежит $\operatorname{supp} \gamma$. Вместе с последним неравенством $h^{\prime}<h$ получаем требуемую цепочку элементов из supp $\gamma$.

Обоснуем теперь основание индукции. Обозначим $\partial \gamma=h_{0} a\left(a \in K^{*}\right)$. Так как $r \gamma=1$ и $h_{0}=v(\gamma)$, то имеем $g_{j} h_{0}=1$ для некоторого $j$. (Иначе по лемме 6 найдется $h^{\prime} \in \operatorname{supp} \gamma$, для которого $h^{\prime}<h_{0}$.) Таким образом, мы получаем равенство $h_{0}=g_{j}^{-1}$.

ТЕОРема 5. Предположим, что $r \in F G, r \neq 0$ и формальные сумми $\gamma, \beta \in \mathscr{F}(G, F)$ таковьи, что $r \gamma=r \beta$. Если $\gamma_{1}, \beta_{1} \in L$ являются началами относительно левого порядка сумм $\gamma$ и $\beta$ соответственно, то либо $\gamma_{1}$ начало $\beta_{1}$, либо, наоборот, $\beta_{1}$ - начало $\gamma_{1}$. В частности, если $\gamma, \beta \in L$, то $\gamma=\beta$.

ДокаЗАтельство. Рассмотрим сечения $\gamma=\gamma_{1}+\delta_{1}, \beta=\beta_{1}+\delta_{2}$. Так как $r(\gamma-\beta)=0$, то либо $\gamma-\beta=0$, либо разность $\gamma-\beta$ не имеет нормы (см. предложение 13). В первом случае утверждение настоящей теоремы очевидно. Разберем второй случай. Заметим сначала, что $\gamma-\beta=\gamma_{1}-\beta_{1}+\delta_{1}-\delta_{2}$. Предположим, что $v\left(\gamma_{1}-\beta_{1}\right)$ не больше ни $\operatorname{supp} \gamma_{1}$, ни $\operatorname{supp} \beta_{1}$. Но $\operatorname{supp} \gamma_{1}<\operatorname{supp} \delta_{1}$ и $\operatorname{supp} \beta_{1}<\operatorname{supp} \delta_{2}$. Отсюда следует, что сушествует элемент $h \in G$, для которого $v\left(\gamma_{1}-\beta_{1}\right) \leqslant h<\operatorname{supp} \delta_{1} \cup \operatorname{supp} \delta_{2}$. Тогда норма $v(\gamma-\beta)$ сушествует и совпадает с нормой $v\left(\gamma_{1}-\beta_{1}\right)$. Получаем противоречие с предположением второго случая. Следовательно, на самом деле норма $v\left(\gamma_{1}-\beta_{1}\right)$ больше либо supp $\gamma_{1}$, либо $\operatorname{supp} \beta_{1}$. Если норма $v\left(\gamma_{1}-\beta_{1}\right)$ больше supp $\gamma_{1}$, то $\gamma_{1}-$ начало ряда $\beta_{1}$, а если $v\left(\gamma_{1}-\beta_{1}\right)>\operatorname{supp} \beta_{1}$, то $\beta_{1}-$ начало ряда $\gamma_{1}$. 
СлеДСТВИЕ. Пусть $r \gamma=1$ u $r \beta=\delta$ для некоторых $\gamma, \beta \in L$. Предположим, что *-произведение $\gamma * \delta$ существует (это так, например, в случае $\delta \in F G)$. Если $\alpha \in L-$ начало $\gamma * \delta$, то $\alpha \prec \beta$. Кроме того, если $\gamma * \delta \in L$, то $\beta=\gamma * \delta$.

ДоКАЗАТЕЛьСТво. Пользуясь ассоциативностью $*$-произведения, получаем: $r(\gamma * \delta)=r \beta$. По теореме 5 либо $\alpha$ - начало ряда $\beta$, либо $\beta$ - собственное начало ряда $\alpha$. Вторая возможность исключается. Действительно, в этом случае $v(\gamma * \delta-\beta)=v(\alpha-\beta) \neq \infty$ и $r(\gamma * \delta-\beta)=0$; применение предложения 13 приводит к противоречию.

Теорема 6. Предположим, что $\gamma \in L \cap R-$ такой степенной ряд, что $r \gamma=1$. Тогда $\gamma r=1$.

ДоКАЗАТЕЛЬСТво. Пусть $\gamma r=1+\delta$. Суммы $\gamma r$ и $\delta=\gamma r-1$ принадлежат $R$, так как $\gamma \in R$. Следовательно,

$$
r=(r \gamma) r=r(\gamma r)=r(1+\delta)=r+r \delta
$$

откуда вытекает равенство $r \delta=0$. Далее, $\delta=0$ согласно предложению 13.

\section{Список литературы}

1. Мальцев А. И. О включении групповых алгебр в алгебры с делением // Докл. АН СССР. 1948. Т. 60. № 9. С. 1499-1501.

2. Дубровина Т. В., Дубровин Н. И. Конусы в группах // Матем. сб. 1996. Т. 187. № 7. C. $59-74$.

3. Кокорин А.И., Копытов В. М. Линейно упорядоченные группы. М.: Наука, 1972.

4. Дубровин Н. И. Обратимость группового кольца правоупорядоченной группы над телом // Матем. заметки. 1987. Т. 42. № 4. С. 508-518.

5. Дубровин Н. И. Рационалњые замыкания групповых колец левоупорядоченных групп // Матем. сб. 1993. Т. 184. № 7. С. 3-48.

6. Дубровин Н.И. Пример цепного первичного кольца с нильпотентными элементами // Матем. сб. 1983. Т. 120 (162). №3. С. 441-447.

Владимирский государственный университет

Поступила в редакцию 24.03.1999 